\title{
A Community Based Cross Sectional Study on Prevalence Of Polycystic Ovarian Syndrome (PCOS) and Health Related Quality of Life in Pakistani Females
}

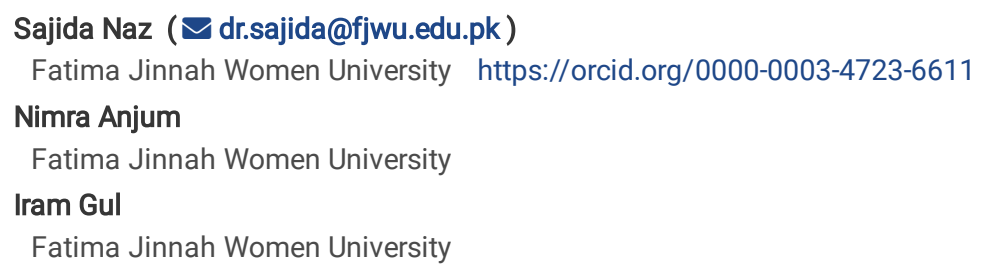




\section{Abstract}

Background: Polycystic Ovarian Syndrome is a hormonal disorder and its symptoms exist in most of young females. In Pakistan, PCOS diagnosis is being reported at an alarming rate, however the frequency of early diagnosis is very low.

Objectives: Objective of this community based study was to find out prevalence of PCOS related symptomology based on Rotterdam criteria in Punjab district of Pakistan. Moreover, the relationship between health status and PCO related quality of life was also assessed.

Methods: 1258 young females within the age range of 18-25 years participated in a cross sectional survey from the various colleges of Punjab District. The participants completed the questionnaire of Short Form-36, Polycystic Ovarian Syndrome Questionnaire and Clinical Profile Form.

Results: There was an increased prevalence of one or two PCOS symptoms (i.e., Menstrual Irregularity and Hirtuism) in most of young females. Most of the females were not aware of the PCOS phenomenon. Furthermore, pearson correlation analysis on study scales revealed that with increase in PCOS symptomology, there are chances that quality of life will be poor.

Discussion and Conclusion: These findings suggest that still most of educated females are largely unaware about PCOS symptoms, it needs further expansion in terms of educational seminars to promote awareness about risk factors. The results of this study can be used by educational institutions or health care centers for promoting PCOS awareness and contributing towards early diagnosis and prevention.

\section{Introduction}

Poly Cystic Ovarian Syndrome (PCOS) is the most frequently diagnosed endocrinal and metabolic disorder around globe which usually affects approximately $7 \%$ of reproductive aged women $(1,2)$. According to World Health Organization, PCOS is an emerging public health issue $(3)$ and most likely to be a lifelong condition which is characterized by unexplained weight gain, menstrual irregularities, facial / body acne and hair though these symptoms may vary from one woman to another (4). Although this condition has not gained much attention, it significantly impacts young women of reproductive age both physically and psychologically. It has been estimated that approximately 4-8\% of the teens and young women suffer from PCO's worldwide (5). PCOS is becoming a frequently diagnosed condition in Pakistan where the prevalence is as high as 15.7-37\% (6, 7). The recent studies conducted in various geographic locations of Pakistan demonstrate that majority of the women are unaware of the symptoms and remain undiagnosed till the symptoms become complicated (8). It has been demonstrated through the clinical reports show that PCOS if untreated, may lead to anovulatory infertility and oligomenorrhoea and other reproductive complications (9).

The etiology of PCOS in women remains unclear, and it has been found to be linked with an-ovulatory infertility menstrual dysfunctions and hirsutism (10). Though this disease is most common and affects every 1 out of 10 women of reproductive age, its etiology is still unclear and diagnostic difficulty remains there. Previous research has suggested that genetic, endocrine, metabolic, environmental and lifestyle are highlighted clinical manifestations of PCOS $(11,12)$. Major etiological factors of PCOS includes an increased alteration in sympathetic nerve activity whichis directly associated with PCOS while women suffering from PCOS exhibit decreased association with parasympathetic nervous activity $(13,14)$.

Difficulty in the diagnosis of PCOS has been reported to be related to the subjective presentation of phenotypes. Literature suggests that PCOS prevalence rate is higher in first degree relatives (15). The prevalence of PCOS in women residing in South Asian regions, particularly in Pakistani is rapidly increasing. According to an estimate, $52 \%$ of the women residing in the Indian subcontinent present with clinically significant symptoms of PCOS (16).

Despite these high figures, there is insufficient published literature that could give insight into the indigenous etiological factors for PCOS in Pakistan. Furthermore, diagnosis of PCOS is source of immense distress for the young females, therefore need for psychoeducation and counseling prevails in midst of fact that majority of the women are not educated enough to identify early signs and symptoms of this disorder. Therefore, this study was first of its kind to look into the perception and attitude towards PCOS in Pakistani young women. This cross sectional study was designed to evaluate prevalence of symptoms of PCOS according to the Rotterdam diagnostic criteria (17). It also looked into the association between PCOS and Health related quality of life in such females

\section{Methods}

\section{Design and Participants}

From February 2019 to August 2019, 1258 female students from various colleges of Rawalpindi, Islamabad, Attock and Lahore (Punjab Districts) participated in a self-report survey related to prevalence of PCOS. Along with the PCOS questionnaire, SF-36 was applied to assess health related quality of life in young women. The presence or absence of acne and androgenic alopecia was recorded, and weight, height, and waist and hip circumferences were alsoassessed. The participants also completed a clinical history form which included details related to menstrual cycle, symptoms of hirsutism, acne, reproductive health, gynecological history, ongoing medications, family history of physical illnesses, and lifestyle habits.Data of Young women who qualified the criteria of PCOS were subjected for further analysis.

The study was approved by the institutional review board of Fatima Jinnah Women University, and written informed consent was obtained from all the participants. The ethical aspects of the present study are covered in the Declaration of Helsinki.

Page 2/8 


\section{Inclusion and exclusion criteria}

Those females who were 18 years and above, with or without history of PCOS related symptoms (and related comorbidity) and who had consented were included in the present study. Those with severe form of physical and psychological disturbance were not included.

\section{Sample Size determination}

The required sample who met eligibility criteria was selected through non-probability purposive sampling technique. Sample size of 1258 was estimated through $\mathrm{G}$ power calculator with $95 \%$ confidence interval and $5 \%$ chance of error (18).

\section{Study tools}

Other than demographic and clinical features of PCO profile, PCOS questionnaire and SF-36 were administered to assess prevalence of PCOS symptomology and the Health Status of the participants. Brief details are as follows:

\section{Demographic and Clinical Profile}

Demographic sheet was prepared to analyze the general characteristics of participants. This sheet consists of questions related to race, socio-economic status, previous education, name of institution and body-mass index though weight and height. These characteristics can inform about association of PCOS patients with their background.

\section{Clinical Profile Form}

Clinical Profile Form was constructed to know the rate of PCOS related symptoms in younger female population. Clinical profile form was prepared on basis of Rotterdam Criteria, used for diagnosing PCOS. According to Rotterdam Criteria, two of the following three symptoms should present for diagnosing PCOS. Criteria includes; Polycystic ovaries, Anovulation and hyperandrogenism. Questions according to Rotterdam criteria were included in profile form for scrutinize the PCOS related symptoms in female youngsters.

\section{Short Form-36:}

Ware and colleagues (19) developed the SF-36 from the Medical Outcomes Study, it is an overall indicator of health Status. Total items are 36 and divided into eight subscales that measure different domains of health-related quality of life. SF-36 includes two component score i-e. physical component score and mental component score. Scores ranges from 0-100. Higher scores on all subscales indicate better health functioning while low scores indicate impairment in health-related quality of life

\section{PCOS Quality of Life Questionnaire (PCOSQ):}

PCOSQ developed by Cronin (20) assess the health-related quality of life in PCOS. It includes 26 items and uses seven-point Likert scale for responses. PCOSQ includes five domains. For each question, 7 score indicates least impairment and 1 score indicates severe impairment in HRQOL

\section{Procedure}

After seeking ethical clearance from the institutional review board, the participants from 10 public sector colleges and universities were approached for the recruitment purpose. According to the clinical standards of sample size estimation, sample size of 1500 was targeted. After the completion of data collection, the data screening procedures were carried out. After scrutiny from possible data entry errors, the final data for computation was 1258 women. The total time to complete the self report questionnaires was 15 minutes. All questionnaires were used in the English language

\section{Data Analysis Procedures}

Combination of descriptive and inferential analysis was used to identify degree of occurrence of PCOS symptomology, health status and health related quality of life. For objective 2, face to face interviews were conducted regarding perception about PCOS. The data was subjected to screening process through with any form of outliers, or missing data was treated before main analysis.

\section{Results}

84\% (1258 out of 1500) women completed the protocols involved in the survey. Study data analysis revealed that both PCOS and SF-36 had appropriate cornbach alpha reliabilities (alpha $=0.82$ and 0.94 respectively). Of the 1258 girls, $165(13.1 \%)$ reported to experience menstrual irregulation, 1101 (87.5\%) had high prevalence of symptoms of Hersuitism and 157 (12.5\%) had both Ml and H. Of the 165 cases, 62 (37.5\%) had undergone hormonal check while 11 (6\%) agreed for the need of ultrasonography. 27 girls (16\%) had already diagnosed PCOS. On the basis of these figures, it can be estimated that if all of the identified cases in this study underwent hormonal assessment, approximately $13 \%-25 \%$ of the young women would have likely to have received diagnosis for PCOS.

Given that there were 165 women who fulfilled PCOS criterion, the prevalence of $3 \%(95 \% \mathrm{Cl} 2.6-4.0)$ in this sample is estimated as per Rotterdam criterion (see Tables 1 to 4 ). Average age range of cases with PCOS was $19.7 \pm 1.71$ years with BMl level of $22 \pm 4.57 \mathrm{Kg} / \mathrm{m}^{2}$, 
Table 1

Demographic Information of the participants $(n=1258)$ in terms of frequency $(\mathrm{f})$, percentages $(\%)$, Mean $(M)$ and Standard Deviation $(S D)$ values

\begin{tabular}{|c|c|c|c|c|}
\hline Variable & Response Category & $f(\%)$ & M & SD \\
\hline \multirow[t]{2}{*}{ Participant Age } & $18-20$ & $538(42.8)$ & & \\
\hline & $21-24$ & 720 (57.2) & 20.78 & 1.56 \\
\hline \multirow[t]{6}{*}{ Education Completed } & F. A & $35(2.8)$ & & \\
\hline & ICS & $91(7.2)$ & & \\
\hline & FSC & $981(78)$ & & \\
\hline & BS & $116(9.2)$ & & \\
\hline & MS & $20(1.6)$ & & \\
\hline & Missing & $5(.39)$ & & \\
\hline \multirow[t]{4}{*}{ Self-Reported SES } & Low & $55(4.4)$ & & \\
\hline & Moderate & $1088(86.5)$ & & \\
\hline & High & $111(8.8)$ & & \\
\hline & Missing & $4(0.3)$ & & \\
\hline \multirow[t]{4}{*}{ Body-Mass Index } & Underweight & $252(20)$ & & \\
\hline & Normal Weight & $601(47.8)$ & & \\
\hline & Overweight & $170(13.5)$ & & \\
\hline & Obese & $225(17.9)$ & & \\
\hline \multirow[t]{2}{*}{ Marital Status } & Un-Married & 1198 (95.2) & & \\
\hline & Married & $60(4.8)$ & & \\
\hline \multirow[t]{3}{*}{ Family System } & Joint & $356(28.3)$ & & \\
\hline & Nuclear & $897(71.3)$ & & \\
\hline & Missing & $5(.4)$ & & \\
\hline
\end{tabular}


Table 2

Pearson's correlation between the sub-domains of Short Form (SF-36) and Polycystic Ovarian Syndrome Questionnaire (PCOSQ) $(\mathrm{n}=1258)$

\begin{tabular}{|c|c|c|c|c|c|c|c|c|c|c|c|c|c|c|}
\hline Variables & 1 & 2 & 3 & 4 & 5 & 6 & 7 & 8 & 9 & 10 & 11 & 12 & 13 & 14 \\
\hline PF & 1 & & & & & & & & & & & & & \\
\hline RP & $.134^{\star}$ & 1 & & & & & & & & & & & & \\
\hline RE & .112 & $.502^{\star \star}$ & 1 & & & & & & & & & & & \\
\hline EF & -.006 & $.192^{\star \star}$ & $.193^{\star \star}$ & 1 & & & & & & & & & & \\
\hline EWB & -.036 & $.277^{\star \star}$ & $.151^{\star}$ & $.517^{\star \star \star}$ & 1 & & & & & & & & & \\
\hline SF & .005 & -.051 & -.078 & .053 & -.008 & 1 & & & & & & & & \\
\hline$P$ & -.031 & $-.298 * \star$ & $-.281^{\star \star}$ & $-.207^{\star \star}$ & $-.280 \star \star$ & $.292^{\star \star}$ & 1 & & & & & & & \\
\hline GH & -.014 & .027 & -.040 & $.182^{\star \star}$ & $.300 \star \star$ & .075 & -.094 & 1 & & & & & & \\
\hline $\mathrm{BH}$ & -.060 & $.334^{\star \star}$ & $.292^{\star \star}$ & $.262^{\star \star}$ & $.404^{\star \star}$ & .035 & $-.293^{\star \star}$ & .077 & 1 & & & & & \\
\hline$E$ & -.050 & $.324^{\star \star}$ & $.309 \star \star$ & $.320 \star \star$ & $.351^{\star \star}$ & .102 & $-.342^{\star \star}$ & .079 & $.718^{\star \star}$ & 1 & & & & \\
\hline W & -.036 & $.413^{\star \star}$ & $.340 \star \star$ & $.263^{\star \star}$ & $.376^{\star \star}$ & .028 & $-.327 \star \star$ & .009 & $.652^{\star \star}$ & $.685^{\star \star}$ & 1 & & & \\
\hline I & .000 & $.277^{\star \star *}$ & $.296^{\star \star}$ & $.326^{\star \star}$ & $.355^{\star \star}$ & $.145^{\star}$ & -.174 *夫 & .069 & $.663^{\star \star *}$ & $.818^{\star \star}$ & $.616^{\star \star}$ & 1 & & \\
\hline M & -.064 & $.319 \star \star$ & $.270 \star \star$ & $.248^{\star \star}$ & $.305^{\star \star}$ & -.011 & $-.355^{\star \star}$ & -.009 & $.670 \star \star$ & $.766^{\star \star}$ & $.688^{\star \star}$ & $.591^{\star \star}$ & 1 & \\
\hline BMI & $.175^{\star \star}$ & -.013 & -.079 & -.011 & $-.129 \star$ & -.042 & -.024 & .032 & -.053 & -.020 & -.222 & -.088 & .033 & 1 \\
\hline \multicolumn{15}{|c|}{ *Correlation is significant at 0.05 level } \\
\hline & & nt & evel & & & & & & & & & & & \\
\hline
\end{tabular}

Note: $P F=$ physical functioning, $R P($ role limitation due to physical functioning), RE (role limitation due to emotional functioning), EF( energy/fatigue), EWB (emotional well-being) ,SF( social functioning), $\mathrm{P}$ (pain), GH (general health), BH (body hair), E (emotions) ,W (weight),I (infertility) , $M$ (menstrual problems) ,BMI (body mass index)

Table 3

Univariate Logistic Analysis of demographic and clinical correlates of $\operatorname{PCOS}(n=1258)$

\begin{tabular}{|lll|}
\hline Variables & OR $(95 \% \mathrm{Cl})$ & $\boldsymbol{p}$ Value \\
\hline Age (yrs.) & $0.64(0.54-1.17)$ & n.s. \\
\hline Income (PKR) & $1.02(1.00-1.19)$ & .03 \\
\hline Family Status & $1.05(1.03-1.13)$ & .02 \\
\hline Weight (Kgs) & $1.06(1.09-1.17)$ & .02 \\
\hline BMl > 23 & $1.16(1.01-1.17)$ & .00 \\
\hline Family History of Type II Diabetes, PCOS & $4.61(1.09-8.76)$ & .00 \\
\hline
\end{tabular}

Table 4

Estimated Prevalence of Cases based on Rotterdam Criteria

\begin{tabular}{|c|c|c|c|c|c|}
\hline Clinical Symptoms of PCOS & $\mathbf{N}$ & $\begin{array}{l}\text { Completed } \\
\text { Evaluation }\end{array}$ & $\begin{array}{l}\text { Confirmed Diagnosis as per } \\
\text { Rotterdam criteria }\end{array}$ & $\begin{array}{l}\text { Probable } \\
\text { cases* }\end{array}$ & $\begin{array}{l}\text { Total exposed to the } \\
\text { condition }\end{array}$ \\
\hline Menstrual Irregularity (MI) & 135 & 62 & 16 & 34 & 42 \\
\hline $\begin{array}{l}\text { Menstrual Irregularity (MI) and } \\
\text { Hirsutism }(\mathrm{H})\end{array}$ & 130 & 11 & 4 & 5 & 7 \\
\hline Total & 165 & 73 & 20 & 39 & 49 \\
\hline
\end{tabular}

\section{Clinical signs and symptoms of PCOS as Assessed through Clinical Profile Form}

Clinical signs and symptoms of PCOS are shown in Fig. 01 using percentage values. According to the responses on Clinical Profile Form, $44 \%$ participants were reported to be aware of PCOS. while $13.2 \%$ of them indicate family history of PCOS. $56 \%$ of the participants reported family history of both Type II Diabetes and PCOS. 32\% of the participants reported partial absence of menses, which is major symptom of PCOS. 32\% of the participants felt difficulty in losing their weight while $25 \%$ reported feeling difficult in performing ADL's (Activities of Daily Living) due to some physical condition. 
During Adolescence, one need extra supplements or vitamin capsules for appropriate growth of body, however in this study only $13 \%$ participants reported take some form of supplements while $86 \%$ relayed on food they ate.

\section{Association between PCOS related Quality of Life and SF-36 (Health Status)}

In Table 2, the results of Pearson's product moment correlation are reported. There was a significant positive correlation between most of the sub-scales (such as Physical Functioning with Role limitation due to Physical Functioning and Emotional Functioning, Physical also shows positive correlation with Emotional Well-Being) except for few. Participants reported positive correlation among Physical Functioning with Role Limitation due to Physical Functioning $(r=.134, p>0.05)$, Role Limitation due to Emotional Functioning $(r=.112)$, Social Functioninng $(r=.005)$ and Body Mass Index $(r=.175$, $p>$ 0.01).

Young females showed positive response on Role limitation due to Emotional Functioning domain with all domains of PCOSQ Scale while three domains of SF-36 Scale; Role Limitation due to Physical Functioning $(r=.502, p>0.01)$, Energy/Fatigue $(r=.193, p>0.01)$, Emotional Well-Being $(r=.151$, $p>0.05)$. Emotional Well-Being domain of SF-36 conveys positive correlation with all the mentioned domains except with Physical Functioning ( $r=-.036)$, Social Functioning ( $r=-.008)$, Pain $(r=-.208, p>0.01)$ and Body Mass Index $(r=-.129, p>0.05)$. Social Functioning domain indicates positive response between Energy/Fatigue (.053), Pain $(r=.292, p>0.01)$ and Infertility $(r=.145, p>0.05)$. Participants reported positive correlation between PCOSQ domains Infertility and Emotions $(r=.818, p>0.01)$, Weight and Menstrual problems $(r=688, p>0.01)$.

\section{Discussion}

PCOS is an under recognized and yet complex and most common condition. Through this study, we have reported perceived clinical features of PCOS in general population of 1258 college going young women. Other than estimation of prevalence rates, we aimed at examining their health status along with presence of PCOS related quality of life and attitudes towards the condition. Most of the women who participated in this study were identified with PCOS symptoms including irregularity in periods (165\% out of 1258). Amongst the different symptoms of PCOS, hirtuism was the most commonly reported. We also observed that despite the high prevalence of these clinical features only $16 \%$ of the women had received proper diagnosis or clinical attention. The remaining \% were either unaware or did not consider it as a significant problem.

As substantiated by the diagnostic literature on PCOS, the most commonly reported presentation of symptoms was ovulatory malfunction, hyperandrogenism, and polycystic

ovarian morphology (PCOM) (21). The metablic features of this condition include predominantly, an abnormality in fat distribution (22). In terms of health status, we found out that the participants who reported high prevalence of clinical features of PCOS had poor health related quality of life on all domains of SF-36. Similar results have been reported by other studies with high correlation between clinical symptoms of PCOS and Quality of Life (23, 24).

There were few limitations of this study, such as diverse sample across various provinces of Pakistan could not be approached Additionally qualitative responses on perception towards PCOS related symptoms can add meaningful insight into contextual factors leading to delay in PCSO recognition and diagnosis among girls.

This study has identified high prevalence of PCOS symptoms among women across various districts of Punjab, Pakistan. As most of the women were either unaware or did not consider the PCOS as a problem, educational programs and clinical awareness is required along with provision of counseling due to expected distress that might be involved. It is pertinent to mention here that in developing countries such as Pakistan, PCOS is either not recognized timely, and the symptoms are likely to be attributed to skin diseases or dermatology, the spread of awareness can lead to timely identification and treatment of the PCOS.

\section{Conclusion And Recommendations}

Significant prevalence of PCOS in general community of Punjab region of Pakistan draws attention towards the issue related to early diagnosis and need for psychoeducation in adolescent girls and families. It is recommended to provide education and counseling to women suffering from the symptoms of PCOS. Long term impact of awareness and education about the PCOS may have immense physical and psychological benefits.

\section{References}

1. Jalilian A, et al. "Prevalence of polycystic ovary syndrome and its associated complications in Iranian women: A meta-analysis.". Iran J Reprod Med. 2015;13(10):591.

2. Landay M, Huang A. and Ricardo Azziz. "Degree of hyperinsulinemia, independent of androgen levels, is an important determinant of the severity of hirsutism in PCOS.". Fertil Steril. 2009;92(2):643-7.

3. Gjönnaess H (1990). A simple treatment for polycystic ovarian syndrome / Halvard Gjönnaess. World health forum 1990; 11(2) : 214-217 https://apps.who.int/iris/handle/10665/52261.

4. Hachey LM, et al. "Clinical Implications of Polycystic Ovary Syndrome in Adolescents.". Nurs Womens Health. 2020;24(2):115-26. 
5. Moran C, Tena G, Moran S, Ruiz P, Reyna R, Duque X. Prevalence of polycystic ovary syndrome and related disorders in Mexican women. Gynecol Obstet Invest. 2010;69(4):274-80.

6. Haq N, et al. Prevalence and Knowledge of Polycystic Ovary Syndrome (PCOS) Among Female Science Students of Different Public Universities of Quetta, Pakistan. Imperial Journal of Interdisciplinary Research. 2017;35(6):385-92.

7. Rizvi, M., Abbas, A., Tanwir, S., Sabah, A., Ali, Z. M., Sundrani, M. M., ... Mir, H.A. (2014). Perception and attitude of patients regarding polycystic ovarian syndrome(PCOS) in tertiary care hospitals of Pakistan-a survey based study. International Journal of Pharmacy \& Therapeutics, 5, 147152.

8. Anjum N, et al. "Prevalence of metabolic syndrome in Pakistani women with polycystic ovarian syndrome.". Pak J Biochem Mol Biol. 2013;46(3):97100.

9. Valdimarsdottir R, et al. Pregnancy and neonatal complications in women with polycystic ovary syndrome in relation to second-trimester antiMüllerian hormone levels. Reproductive BioMedicine Online. 2019;39(1):141-8.

10. Chapman JC, et al. "The estrogen-injected female mouse: new insight into the etiology of PCOS." Reprod. Biol Endocrinol. $2009 ; 7(1): 47$.

11. Rutkowska AZ, Diamanti-Kandarakis E. "Polycystic ovary syndrome environmental toxins" Fertil Steril. 2016;106(4):948-58.

12. Tekin G, et al. Altered autonomic neural control of the cardiovascular system in patients with polycystic ovary syndrome. Int $\mathrm{J}$ Cardiol Heart Vasc. 2008;130(1):49-55.

13. Sverrisdottir Y, Bergmann, et al. Is polycystic ovary syndrome associated with high sympathetic nerve activity and size at birth? Am J Physiol Endocrinol Metab. 2008;294(3):E576-81.

14. Raperport C. and Roy Homburg. "The source of polycystic ovarian syndrome.". Clin Med Insights Reprod Health. 2019;13:1179558119871467.

15. Chae J, et al. Metabolic features of adult and adolescent first-degree relatives of women with polycystic ovary syndrome: a systematic review and meta-analysis. Fertil Steril. 2017;108(3):e248-9.

16. Bharathi R, Vidya, et al. An epidemiological survey: Effect of predisposing factors for PCOS in Indian urban and rural population. Middle East Fertil Soc J. 2017;22(4):313-6.

17. Mumusoglu S, Bulent Okan Yildiz. "PCOS phenotypes and prevalence: Differential impact of diagnostic criteria and clinical versus unselected population." Curr Opin Endocr Metab Res (2020).

18. Faul F, et al. G* Power 3: A flexible statistical power analysis program for the social, behavioral, and biomedical sciences. Behav Res Methods. 2007;39(2):175-91.

19. Ware JE Jr, Cathy Donald S. "The MOS 36-item short-form health survey (SF-36): I. Conceptual framework and item selection." Medical care (1992): 473-483.

20. Cronin L, et al. "Development of a health-related quality-of-life questionnaire (PCOSQ) for women with polycystic ovary syndrome (PCOS).". Int J Clin Endocrinol Metab. 1998;83(6):1976-87.

21. Escobar-Morreale HF. Polycystic ovary syndrome: definition, aetiology, diagnosis and treatment. Nat Rev Endocrinol. 2018;14(5):270.

22. Boumosleh J, Matar, et al. Metabolic concomitants of obese and nonobese women with features of polycystic ovarian syndrome. J Endocr Soc. 2017;1(12):1417-27.

23. Panico A, et al. Quality of life in overweight (obese) and normal-weight women with polycystic ovary syndrome. Patient Prefer Adherence. 2017;11:423.

24. Moghadam Z, Behboodi, et al. Measures of health-related quality of life in PCOS women: a systematic review. Int J Womens Health. $2018 ; 10: 397$.

\section{Figures}




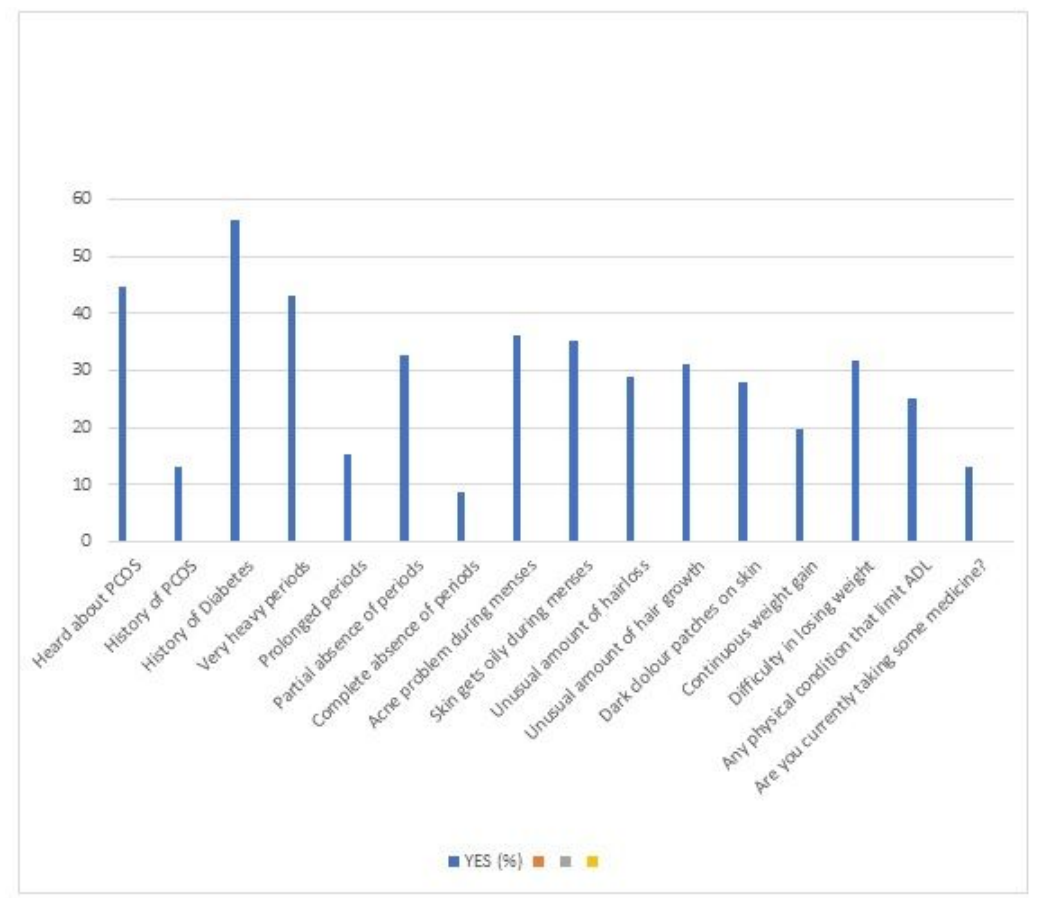

Figure 1

Showing percentage of Yes responses on Clinical Profile Form $(n=1258)$ 